\title{
A comparison between Belsey Mark IV and laparoscopic Nissen fundoplication in patients with large paraesophageal hernia
}

\author{
Danuel V. Laan, MD, ${ }^{\mathrm{a}}$ John Agzarian, MD, ${ }^{\mathrm{a}}$ William S. Harmsen, MS, ${ }^{\mathrm{b}}$ K. Robert Shen, MD, ${ }^{\mathrm{a}}$ \\ Shanda H. Blackmon, MD, ${ }^{\mathrm{a}}$ Francis C. Nichols III, MD, ${ }^{\mathrm{a}}$ Stephen D. Cassivi, MD, ${ }^{\mathrm{a}}$ Dennis A. Wigle, MD, ${ }^{\mathrm{a}}$ \\ and Mark S. Allen, MD
}

\section{ABSTRACT}

Objectives: Laparoscopic Nissen fundoplication is the most commonly performed operation for the repair of large hiatal hernias. We compared outcomes between the Belsey Mark IV fundoplication and the laparoscopic Nissen fundoplication.

Methods: A retrospective review was performed over a 10-year period on patients who had repair of large paraesophageal hernia. Patients who received the Belsey Mark IV $(\mathrm{n}=118)$ were matched 1 to 1 , by year of surgery, gender, and age, with patients who received laparoscopic Nissen fundoplication. We compared these 2 groups, examining recurrence, need for reoperation, perioperative outcomes, and symptomatic follow-up as defined by the Gastroesophageal Reflux DiseaseHealth Related Quality of Life questionnaire.

Results: Recurrence rates were similar between patients who had a Belsey Mark IV and laparoscopic Nissen fundoplication $(8.4 \%$ vs $16.1 \%, P=.11)$. However, the esophageal leak rate was higher in patients who received a laparoscopic Nissen fundoplication compared with the Belsey Mark IV (6.8\% vs $0 \%$, respectively, $P=.006)$, and patients who received a laparoscopic Nissen fundoplication had higher rates of reoperation ( $9.3 \%$ vs $2.5 \%$, respectively, $P=.05$ ). Gastroesophageal Reflux Disease-Health Related Quality of Life symptom scores were similar between groups with symptoms in laparoscopic Nissen fundoplication and Belsey Mark IV, being excellent $(74.4 \%$ vs $81.4 \%)$, good $(9.3 \%$ vs $7.0 \%)$, fair $(9.3 \%$ vs $0)$, and poor $(7.0 \%$ vs $11.6 \%)$, respectively $(P=.52)$.

Conclusions: Laparoscopic Nissen fundoplication for large paraesophageal hernias was associated with an increased incidence of leak and reoperation when compared with Belsey fundoplication. Belsey Mark IV fundoplication should be considered when deciding on what operation to perform in patients with large paraesophageal hernias. (J Thorac Cardiovasc Surg 2018;156:418-28)

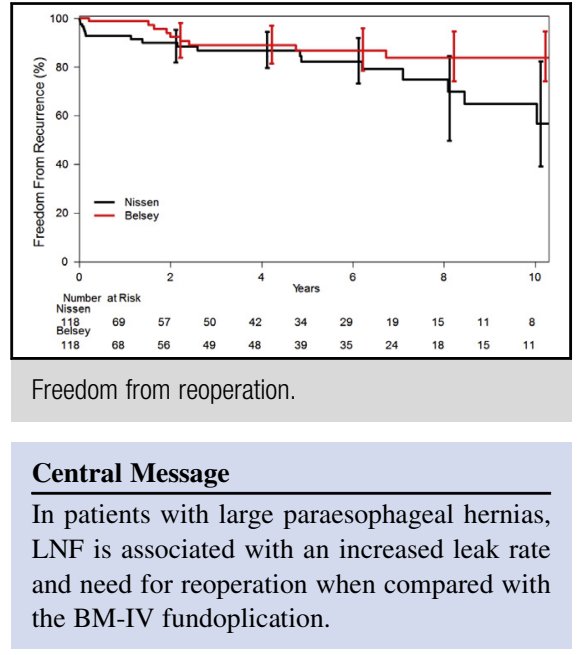

\section{Perspective}

LNF is the most commonly performed operation for symptomatic large paraesophageal hernias. Our study shows that laparoscopic Nissen fundoplication is associated with higher rates of postoperative leaks and need for reoperation. Further investigation is needed to develop a tailored approach to the patient with a large paraesophageal hernia.

See Editorial Commentary page 429.
The most commonly performed surgical treatment for symptomatic hiatal hernia refractory to medical therapy is

From the ${ }^{\mathrm{a}}$ Division of General Thoracic Surgery, Department of Surgery, and ${ }^{\mathrm{b}}$ Department of Biomedical Statistics and Informatics, Mayo Clinic, Rochester, Minn. Read at the 97th Annual Meeting of The American Association for Thoracic Surgery, Boston, Massachusetts, April 29-May 3, 2017.

Received for publication April 28, 2017; revisions received Nov 7, 2017; accepted for publication Nov 20, 2017; available ahead of print Feb 1, 2018.

Address for reprints: Danuel V. Laan, MD, Division of General Thoracic Surgery, Mayo Clinic, 200 First St, SW, Rochester, MN 55905 (E-mail: laan.danuel@ mayo.edu).

0022-5223/\$36.00

Copyright (c) 2017 by The American Association for Thoracic Surgery

https://doi.org/10.1016/j.jtcvs.2017.11.092 the laparoscopic Nissen fundoplication (LNF). The laparoscopic approach offers several advantages compared with its open counterparts, accounting for its increased use. ${ }^{1,2}$ These include shorter hospital length of stay, reduced postoperative pain, and reproducibility of results compared with transthoracic Belsey Mark IV (BM-IV)

Scanning this QR code will take you to the article title page. To view the AATS Annual Meeting Webcast, see the URL next to the webcast thumbnail.

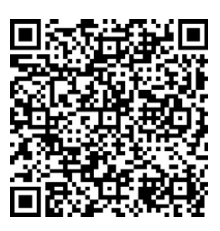




\section{Abbreviations and Acronyms \\ BMI = body mass index \\ BM-IV $\quad=$ Belsey Mark IV \\ $\mathrm{CD}=$ Clavien-Dindo \\ CI $\quad=$ confidence interval \\ CT $=$ computed tomography \\ EGD = esophagogastroduodenoscopy \\ GERD = gastroesophageal reflux disease \\ GERD-HRQL $=$ Gastroesophageal Reflux Disease- Health Related Quality of Life \\ HR = hazard ratio \\ LNF = laparoscopic Nissen \\ fundoplication}

fundoplication. However, recent reports have demonstrated recurrence rates of $15 \%$ to $25 \%$ after laparoscopic repair of large hiatal hernias. 3,4

Rudolph Nissen ${ }^{5,6}$ proposed a fundoplication of the stomach via an abdominal approach in 1955. Many modifications to the original Nissen procedure have been made that have brought it to its current form, including reapproximation of the hiatus, division of the short gastric vessels, and creation of a loose, floppy wrap $2 \mathrm{~cm}$ long. ${ }^{7,8}$ Concurrently, Sir Ronald Belsey developed an intrathoracic fundoplication, the Belsey procedure. Over the course of 20 years, Belsey made 3 key modifications involving the degree of fundoplication of the stomach. His final procedure was termed the "BM-IV fundoplication" to signify this stepwise development. In patients with a foreshortened esophagus, an esophageal lengthening procedure (Collis gastroplasty) was added as an option to both procedures due to contributions by J. Leigh Collis and later Griffith Pearson, Robert Henderson, and Mark Orringer. ${ }^{9}$ The laparoscopic counterpart to this is the wedge gastroplasty.

Wide adoption of the BM-IV procedure occurred primarily after a report by Skinner and Belsey that showed an $85 \%$ success rate in a review of 600 patients in $1961 .^{10,11}$ In the laparoscopic era, however, this procedure has fallen out of favor because it can only be performed transthoracically. Although the BM-IV is not widely performed, it is a favorable choice in patients with extreme obesity, large hiatal hernias, previous abdominal surgery, redo surgery, esophageal dysmotility, and extreme esophageal shortening. ${ }^{12}$

In contrast to patients with gastroesophageal reflux from a small hiatal hernia, symptoms from a large paraesophageal hernia (at least $50 \%$ of the stomach in the chest) are more related to obstruction and include chest and epigastric pain, dysphagia, shortness of breath, early satiety, and anemia secondary to bleeding from Cameron erosions (ulcers that arise from the stomach rubbing on the diaphragmatic crus). The incidence of gastroesophageal reflux disease
(GERD) in large paraesophageal hernias is a debated topic. Some reports have found the incidence to be low, ${ }^{13,14}$ whereas others have found the incidence to be high and have postulated the development of large paraesophageal hernias to be a continuum of a sliding hernia. ${ }^{15}$

In a previous review of patients with intrathoracic stomach treated at our institution we found a tendency toward increased leak and complications in patients treated with open Nissen fundoplication and uncut Collis-Nissen fundoplication through the chest when compared with the BM-IV; however, symptoms were similar at a median follow-up of 42 months. This initial study was limited, in that only $17 \%$ of patients in that cohort had Nissen repairs without an uncut Collis and none were via a laparoscopic technique. $^{16}$

Reported advantages to the BM-IV are many: It allows a barrier to reflux while maintaining normal gullet functions (ie, normal swallowing, belching, and vomiting), extensive mobilization of the esophagus, and concomitant procedures to the chest wall, lung, and esophagus. However, in the modern era, little attention has been directed toward the BM-IV as a means of primary repair for patients with large paraesophageal hernias. Likewise, no retrospective studies have compared the BM-IV with LNF in this specific group of patients. As such, the goal of our study was to compare the outcomes between the BM-IV fundoplication and the LNF.

\section{PATIENTS AND METHODS}

We performed a retrospective review of a prospectively collected institutional database, including all patients from 2002 to 2011 who had repair of a large paraesophageal hernia (defined as $\geq 50 \%$ of the stomach within the thorax as seen on a preoperative barium study). Hernia size was determined by preoperative esophagram and correlated with intraoperative findings. Exclusion criteria included patients with no clinical follow-up posthospital discharge ( $\mathrm{n}=3$ in Belsey, $\mathrm{n}=28$ in Nissen); operations for recurrent hiatal hernias after prior attempted repair regardless of approach $(\mathrm{n}=27)$; hiatal hernia repairs other than LNF or BM-IV (open abdominal Nissen fundoplication $(\mathrm{n}=441)$, Hill fundoplication $(\mathrm{n}=8)$, anatomic repair $(n=14)$, Toupet fundoplication $(n=36)$, or Dor fundoplication $(\mathrm{n}=20)$ ); and patients who had any operation for sliding hiatal hernias, esophageal motility disorders, or achalasia $(n=524)$. All patients who had a BM-IV $(\mathrm{n}=118)$ were matched 1 to 1 with patients undergoing LNF. Matching variables included year of surgery \pm 1 year, gender, and age \pm 1 year. Last clinical follow-up date was defined as the date of last evaluation by a thoracic surgeon, gastroenterologist, or primary care provider for multisystem evaluation. Current symptoms were assessed using the Gastroesophageal Reflux Disease-Health Related Quality of Life (GERD-HRQL) questionnaire $^{17}$ at the time of this study. Patients were contacted by phone, and if they were unreached after 2 attempts, a mail-out questionnaire was sent. Raw GERD-HRQL scores were converted to categoric variables as follows: excellent: 0 to 5; good: 6 to 10 ; fair: 11 to 15 ; and poor: 16 or greater.

The primary outcomes included recurrence and need for reoperation. Patients were evaluated for recurrence using esophagram, esophagogastroduodenoscopy (EGD), or computed tomography (CT) scan. Recurrence was noted if the study demonstrated greater than physiologic herniation of the gastroesophageal junction above the diaphragmatic hiatus $(\geq 2 \mathrm{~cm})$, a slipped wrap, or a disrupted wrap. Expected postwedge 
gastroplasty radiographic or endoscopic features were not reported as recurrences. Secondary outcomes included intraoperative and postoperative complications and postrepair symptoms scoring. Postoperative complications were defined using the Clavien-Dindo (CD) Scoring Model. ${ }^{18}$

\section{Statistical Analysis}

Descriptive statistics are reported as mean ( \pm standard deviation) for continuous variables and as number (percentage) for discrete variables. Follow-up duration is reported as median and range. Patient demographics were compared between the Belsey and Nissen groups using a 2-sample $t$ test for continuous variable or Fisher exact test for categoric data. Risk of recurrence and reoperation was assessed using a univariate Cox model. The results are reported as hazard ratio (HR) and $95 \%$ confidence interval (CI). The alpha-level of 0.05 was set for statistical significance. SAS (SAS Institute Inc, Cary, NC) version 9.4 was used for all analyses.

\section{RESULTS}

From 2002 to 2011, more than 1500 hiatal hernia repairs were performed at our institution. A total of 643 patients $(42 \%)$ had the BM-IV or LNF for treatment of paraesophageal hernia (Figure 1). This group was composed of patients with small paraesophageal hernias $(<50 \%$ of the stomach within thorax, $\mathrm{n}=80)$, moderate paraesophageal hernias $(\geq 50 \%$, but $<75 \%$, of the stomach within the thorax, $\mathrm{n}=246$ ), and intrathoracic upside-down stomach $(\geq 75 \%$ of stomach within the thorax, $\mathrm{n}=317)$. Among patients with moderate paraesophageal hernias or intrathoracic stomach $(\mathrm{n}=563)$, BM-IV was performed in $121(21.5 \%)$ and
LNF in $442(78.5 \%)$. Median clinical follow-up was 959 days (range, 7-5257 days) in the LNF cases and 657 days (range, 6-5292 days) in the Belsey group. Six surgeons performed the BM-IV during the study period, but the majority of operations ( $94 \%$ ) were performed by a single surgeon in the group (M.S.A.). When BM-IV was performed by surgeons other than M.S.A., it was performed in the setting of increased abdominal adiposity and when a patient had a significant history of multiple previous abdominal procedures. With the exception of 1 surgeon (M.S.A.), who prefers the BM-IV in patients with large paraesophageal hernia, LNF was performed by all surgeons within the group with relative equal distribution. A total of $118 \mathrm{BM}-\mathrm{IV}$ cases were matched to LNF cases. At the time of this study, $83 \%(n=99)$ of patients who received the BM-IV and 79\% $(n=94)$ of patients who received the LNF were alive. Patient demographics, symptoms at time of presentation, and results of preoperative esophagram, EGD, and esophageal motility study are shown in Table 1 . Intrathoracic stomach $(\geq 75 \%$ of stomach within the thorax) was present in all $118(100 \%)$ of the BM-IV cases versus $53(44.9 \%)$ of Nissen cases $(P<.0001)$. Moderate paraesophageal hernia was present in the remainder of LNF cases $(\mathrm{n}=65,55.1 \%)$. Eight patients $(6.8 \%)$ who received the BM-IV and 5 patients $(4.2 \%)$ who received the LNF had urgent repair because of acute obstructive or bleeding

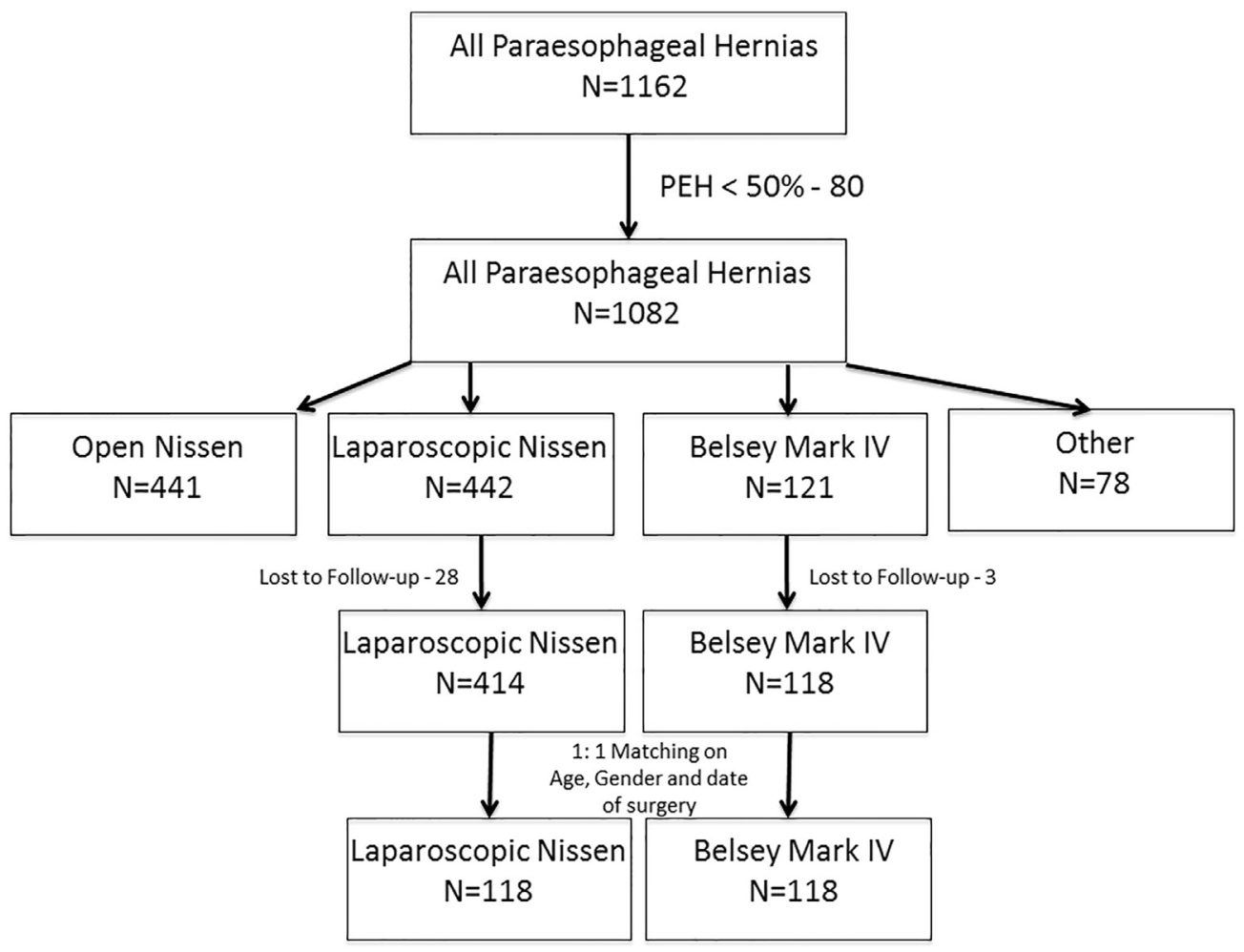

FIGURE 1. Consort diagram of the patient distribution. $P E H$, Paraesophageal hernia. 
TABLE 1. Patient demographics and preoperative characteristics

\begin{tabular}{|c|c|c|c|}
\hline & $\begin{array}{c}\text { Belsey } \\
\mathrm{n}=118\end{array}$ & $\begin{array}{c}\text { Nissen } \\
\mathbf{n}=\mathbf{1 1 8}\end{array}$ & $P$ value \\
\hline Age, median (range), $y^{*}$ & $71.0(37.6-86.4)$ & $71.9(38.2-88.2)$ & $*$ \\
\hline Men & $35(29.7 \%)$ & $35(29.7 \%)$ & * \\
\hline BMI, mean (SD) & $30.3(5.0)$ & $28.8(4.7)$ & .04 \\
\hline No. of prior abdominal surgeries, mean (SD) & $0.9(0.9)$ & $0.7(0.9)$ & .22 \\
\hline \multicolumn{4}{|l|}{ Symptoms at presentation } \\
\hline Obstructive & $99(83.8 \%)$ & $90(76.2 \%)$ & .19 \\
\hline Respiratory & $5(4.2 \%)$ & $8(6.8 \%)$ & .39 \\
\hline Anemia & $9(7.6 \%)$ & $10(8.5 \%)$ & .81 \\
\hline Reflux & $5(4.2 \%)$ & $10(8.5 \%)$ & .18 \\
\hline Preoperative esophagram, yes & 118 & 118 & \\
\hline Preoperative EGD, yes & $110(93.2 \%)$ & $114(96.6 \%)$ & .40 \\
\hline No esophagitis & $97(82.2 \%)$ & $102(86.4)$ & \\
\hline Grade A esophagitis $\dagger$ & $11(9.3 \%)$ & $7(5.9 \%)$ & \\
\hline Grade B esophagitis & $2(1.7 \%)$ & 0 & \\
\hline Grade C esophagitis & 0 & $2(1.7 \%)$ & \\
\hline Grade D esophagitis & 1 & $2(1.7 \%)$ & \\
\hline Barrett's esophagus & $10(8.5 \%)$ & $5(4.2 \%)$ & .16 \\
\hline Cameron erosions & $24(20.3 \%)$ & $23(19.5 \%)$ & .57 \\
\hline Preoperative motility study, yes & $19(16.1 \%)$ & $16(13.6 \%)$ & .22 \\
\hline Normal & $4(3.4 \%)$ & $7(5.9 \%)$ & \\
\hline Technically limited & $11(9.3 \%)$ & $5(4.2 \%)$ & \\
\hline Shortened esophagus & $2(1.7 \%)$ & 0 & \\
\hline Diminished LES tone & $2(1.7 \%)$ & $4(3.4 \%)$ & \\
\hline
\end{tabular}

$B M I$, Body mass index; $S D$, standard deviation; $E G D$, esophagogastroduodenoscopy; $L E S$, lower esophageal sphincter. *A variable used as matching criteria. $\dagger$ Los Angeles Grading System for Esophagitis. ${ }^{24}$

symptoms $(P=.57)$. All other patients received elective repair. For patients who had LNF, $80(67.8 \%)$ had a wedge gastroplasty; no patient who had a BM-IV had an esophageal lengthening procedure. The rate of wedge gastroplasty for patients receiving LNF by year at our institution during the study period $(2002-2011)$ was $18 \%, 63 \%, 84 \%, 74 \%, 75 \%, 72 \%, 61 \%, 61 \%, 54 \%$, and $69 \%$, respectively. Intraoperative complications occurred in 0 BM-IV cases and $2.5 \%(\mathrm{n}=3)$ LNF cases $(P=.12)$. Intraoperative complications included colon laceration, pneumothorax requiring tube thoracostomy, and hemorrhage requiring conversion to laparotomy for hemorrhage control. No patient in either cohort had mesh reinforcement of the hiatus.

Postoperative characteristics are summarized in Table 2. Major (grade III-V) or minor (Grade I-II) morbidity as defined by the $\mathrm{CD}$ grading system were similar between groups. However, patterns of complications differed in that leak from the gastroesophageal junction was higher in the LNF group versus the BM-IV group (8 [6.8\%] vs 0 , respectively; $P=.006$ ). Of the patients with leak, 5 $(62.5 \%)$ had LNF with wedge gastroplasty and $3(37.5 \%)$ had LNF without wedge gastroplasty $(5 / 80[6.25 \%]$ had leaks with wedge gastroplasty, and 3/38 [7.8\%] had leaks without wedge gastroplasty). Complication subtypes are defined in Table 3. Other than leak, there was no statistical difference in the rate of any complication between the 2 groups.

Postoperative dysphagia was observed in 7.6\% $(\mathrm{n}=9)$ of LNF cases and $2.5 \%(\mathrm{n}=3)$ in BM-IV cases $(P=.14)$. Post-thoracotomy pain requiring pain specialty consult was observed in 1 patient in the BM-IV group. There was only 1 perioperative death, and this occurred in the LNF group. On the second postoperative day, this patient had pulseless electrical activity and was resuscitated and transferred to the intensive care unit. Anoxic brain injury occurred, and the patient died on postoperative day 5. Median time to a postfundoplication soft diet was 1 day in the LNF group and 3 days in the BM-IV group. Median length of stay was 3 days (range, 1-68) in the LNF group and 6 days (range, 4-42) in the BM-IV group $(P=.05)$.

\section{Recurrence and Reoperation}

Follow-up studies included esophagram ( $\mathrm{n}=84$ in BM$\mathrm{IV}, \mathrm{n}=78$ in LNF), EGD $(\mathrm{n}=12$ in BM-IV, $\mathrm{n}=19$ in LNF $)$, and CT scan $(\mathrm{n}=9$ in BM-IV, $\mathrm{n}=16$ in LNF). Recurrence was noted on esophagram ( $n=9$ in BM-IV, $n=12$ in LNF), $\operatorname{EGD}(\mathrm{n}=8$ in BM-IV, $\mathrm{n}=9$ in LNF), and CT $\operatorname{scan}(\mathrm{n}=0$ in 
TABLE 2. Operative and postoperative characteristics

\begin{tabular}{|c|c|c|c|}
\hline & $\begin{array}{c}\text { Belsey } \\
\mathrm{n}=118\end{array}$ & $\begin{array}{c}\text { Nissen } \\
\mathrm{n}=118\end{array}$ & $P$ value \\
\hline $\begin{array}{l}\text { Paraesophageal hernia size } \\
\text { Intrathoracic stomach }(\geq 75 \%) \\
\text { Moderate }(50 \%-75 \%)\end{array}$ & $\begin{array}{c}100 \\
0\end{array}$ & $\begin{array}{l}53(44.9 \%) \\
65(55.1 \%)\end{array}$ & $<.0001$ \\
\hline OR time (min), median (range) & $202.5(132-258)$ & $202.0(95-275)$ & .85 \\
\hline Intraoperative complications* & 0 & $3(2.5 \%)$ & .12 \\
\hline Length of stay (d), median (range) & $6(4-42)$ & $3(1-68)$ & .05 \\
\hline $\begin{array}{l}\text { CD Score } \\
\quad<3 \\
\geq 3\end{array}$ & $\begin{array}{c}21(17.8 \%) \\
4(3.4 \%)\end{array}$ & $\begin{array}{l}13(11.0 \%) \\
13(11.0 \%)\end{array}$ & .67 \\
\hline Leak, yes & 0 & $8(6.8 \%)$ & .006 \\
\hline $\begin{array}{l}\text { Follow-up study } \\
\text { Esophagram } \\
\text { EGD } \\
\text { CT scan }\end{array}$ & $\begin{array}{l}85(72.0 \%) \\
13(11.0 \%) \\
11(9.3 \%)\end{array}$ & $\begin{array}{l}77(65.3 \%) \\
19(16.1 \%) \\
16(13.5 \%)\end{array}$ & \\
\hline Follow-up d, median (range) & $667(6-5257)$ & $959.5(7-5292)$ & \\
\hline
\end{tabular}

$O R$, Operating room; $C D$, Clavien-Dindo; $E G D$, esophagogastroduodenoscopy; $C T$, computed tomography. *Intraoperative complications: colon laceration, pneumothorax requiring tube thoracostomy, and hemorrhage requiring conversion to laparotomy for hemorrhage control.

M-IV, $\mathrm{n}=5$ in LNF). Patients with recurrence often had more than 1 study confirming the presence of recurrence. Follow-up esophagram, EGD, or CT scan was not obtained in $19 \%(n=23)$ of the BM-IV group and $18 \%(n=22)$ of the LNF group. Median time to study was 54.5 days (range, 6-4081 days) in the BM-IV group and 46 days (range, 7-3673 days) in the LNF group. Routine postoperative esophagram was obtained less than 90 days after operation in $54 \%(n=64)$ of the BM-IV group and $46 \%(n=54)$ of the LNF group. Esophagram, EGD, or CT scan was obtained more than 90 days postoperatively in $26 \%$ $(n=31)$ of the BM-IV group and $35 \%(n=42)$ of the LNF group on the basis of new or persistent symptoms or for surveillance of gastrointestinal pathology. In total, any recurrence was noted in $10(8.4 \%)$ of the patients in the

TABLE 3. Postoperative complications

\begin{tabular}{lccc}
\hline & $\begin{array}{c}\text { Belsey } \\
\mathbf{n}=\mathbf{1 1 8}\end{array}$ & $\begin{array}{c}\text { Nissen } \\
\mathbf{n}=\mathbf{1 1 8}\end{array}$ & $\boldsymbol{P}$ value \\
\hline Recurrence & $10(8.4 \%)$ & $19(16.1 \%)$ & .11 \\
Reoperation & $3(2.5 \%)$ & $11(9.3 \%)$ & .05 \\
Leak & 0 & $8(6.8 \%)$ & .006 \\
UTI/urinary retention & $3(2.5 \%)$ & $6(5.1 \%)$ & .31 \\
Atrial arrhythmia & $6(5.1 \%)$ & $3(2.5 \%)$ & .31 \\
Respiratory complications & $5(4.2 \%)$ & $2(1.7 \%)$ & .47 \\
Myocardial infarction/PE & $1(0.9 \%)$ & $1(0.9 \%)$ & 1.0 \\
Ileus & $4(3.4 \%)$ & $1(0.9 \%)$ & .37 \\
\hline Death & 0 & $1(0.9 \%)$ & 1.0 \\
Chylothorax & $1(0.9 \%)$ & 0 & 1.0 \\
\hline
\end{tabular}

UTI, Urinary tract infection; PE, pulmonary embolism.
BM-IV group and $19(16.1 \%)$ of the patients in the LNF group $(P=.11)$. Types of recurrence included reherniation ( $\mathrm{n}=7$ in BM-IV, $\mathrm{n}=17$ in LNF), disrupted wrap $(\mathrm{n}=2$ in BM-IV, $n=1$ in LNF), and a slipped wrap ( $n=1$ in BM-IV, $\mathrm{n}=1$ in LNF). Among the LNF cohort, recurrence was noted in $11 \%(\mathrm{n}=9)$ of patients who had wedge gastroplasty $(\mathrm{n}=80)$ and $26 \%(\mathrm{n}=10)$ of patients who $\operatorname{did} \operatorname{not}(\mathrm{n}=38)$. A univariate cox model analysis for factors potentially associated with recurrence (BMI, age, prior abdominal surgery, urgent repair, and performance of a wedge gastroplasty) was done (Table 4). This revealed that the performance of a wedge gastroplasty was protective of recurrence. There was no significant association of BMI, age, prior abdominal surgery, or urgent repair and risk of recurrence. No multivariable model was possible because of limited significant factors on univariate analysis. Kaplan-Meier estimate of 10-year freedom from recurrence

TABLE 4. Risk factors for recurrence

\begin{tabular}{lccc}
\hline & HR & $\mathbf{9 5} \%$ CI & $\boldsymbol{P}$ value \\
\hline BMI, per 1 point & 1.02 & $0.94-1.10$ & .68 \\
BMI category & & & \\
$\quad 25-30$ & 1.79 & $0.51-6.36$ & .37 \\
$\quad 30-35$ & 0.93 & $0.22-3.89$ & .92 \\
$\quad$ >35 & 2.69 & $0.67-10.82$ & .17 \\
Age, per 10 y & 0.89 & $0.67-1.19$ & .44 \\
Prior abdominal surgery, yes & 1.19 & $0.57-2.51$ & .63 \\
Urgent, yes & 0.52 & $0.03-9.56$ & .66 \\
Wedge gastroplasty, yes & 0.38 & $0.15-0.94$ & .03 \\
\hline$H R$, Hazard ratio; $C I$, confidence interval; $B M I$, body mass index. &
\end{tabular}

$H R$, Hazard ratio; $C I$, confidence interval; $B M I$, body mass index 


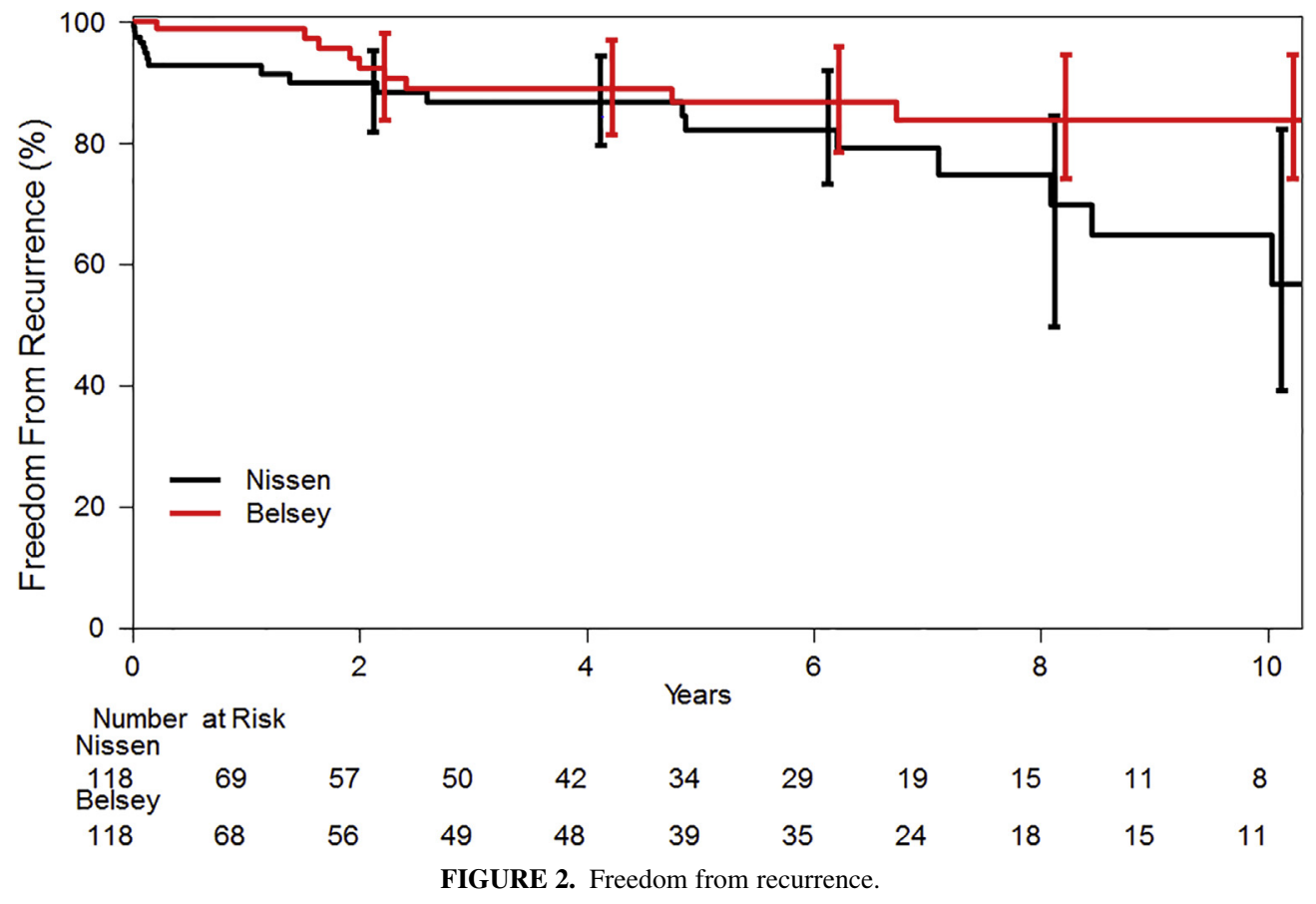

in the BM-IV group was $83.75 \%$ (95\% CI, 69.33-94.58) compared with $64.79 \%(95 \% \mathrm{CI}, 46.58-84.40)$ in the LNF group, corresponding with a nonsignificant increased risk of recurrence in the LNF group (HR, 2.1; 95\% CI, 0.97-4.54; $P=.06$ ) (Figure 2).

In total, reoperation occurred in $2.5 \%(\mathrm{n}=3)$ of the BMIV group and $9.3 \%(\mathrm{n}=11)$ of the LNF group $(P=.05)$. Of the patients in the BM-IV group who required reoperation $(\mathrm{n}=3)$, all were for symptomatic recurrence. Repeat BMIV was performed in 2 patients, and open abdominal Nissen fundoplication was performed in 1 patient. No patients in the BM-IV group required reoperation for perforated viscous. Of the patients in the LNF group who required reoperation $(\mathrm{n}=11), 5(45.5 \%)$ were for leak or necrotic bowel (esophagogastrectomy, $\mathrm{n}=2$ ), partial gastrectomy with Dor fundoplication $(\mathrm{n}=1)$, repair of perforated bowel $(\mathrm{n}=2), 5$ $(45.5 \%)$ were for repair of symptomatic recurrence (repeat Nissen via laparotomy, $\mathrm{n}=2$ ), Dor fundoplication $(\mathrm{n}=2)$, and reduction of recurrence with gastropexy $(\mathrm{n}=1)$, and 1 patient had negative exploratory laparotomy for suspected leak. Kaplan-Meier estimate of 10-year freedom from reoperation for the BM-IV group was 95.6\% (95\% CI, 85.2-100) compared with $85.8 \%$ (95\% CI, 72.5-95.5) in the LNF group corresponding with a significantly increased risk of reoperation in those receiving LNF (HR, 3.7; 95\% CI, 1.0-13.3; $P=.04$ ) (Figure 3).

Of the patients still living at the time of this study $(83 \%$ [n = 99] of BM-IV and 79\% [n=94] of LNF), $43 \%$ $(n=43)$ of the BM-IV group and $45 \%(n=43)$ of the LNF group responded to the GERD-HRQL questionnaire.
Median time from surgery to responding to the questionnaire was similar between groups (9.6 years in BM-IV group vs 10.6 years in LNF group, $P=.66$ ). GERD-HRQL symptoms scores were similar between groups with symptoms in LNF and BM-IV being excellent $(74.4 \%$ vs $81.4 \%)$, good $(9.3 \%$ vs $7.0 \%)$, fair $(9.3 \%$ vs 0$)$, and poor $(7.0 \%$ vs $11.6 \%)$, respectively $(P=.52)$. There was no difference in postoperative use of acid-reducing medication for responders to the questionnaire $(37.2 \%$ $[\mathrm{n}=16]$ in $\mathrm{LNF}$ and $48.4 \%[\mathrm{n}=21]$ in BM-IV, $P=.27)$. There was no difference in overall patient satisfaction with their current gastrointestinal health between LNF or BM-IV groups, with those reporting satisfied ( $72 \%$ in LNF vs $83 \%$ in BM-IV), neutral ( $16 \%$ in LNF vs $2 \%$ in BM-IV), or dissatisfied ( $12 \%$ in LNF vs $14 \%$ in BM-IV), $P=.29$. There was no difference between responders and nonresponders with regard to median age (69 vs 71 years, $P=.25$ ), male gender $(48 \%$ vs $51 \%$, $P=.49$ ), median BMI (30 vs $29.4, P=.63$ ), intraoperative complications $(1.2 \%$ vs $2.4 \%)$, or length of stay (5 vs 6 days, $P=.08)$, respectively.

\section{DISCUSSION}

Patients with paraesophageal hernia accounted for approximately half of patients treated surgically for hiatal hernia at our institution over a 10 -year period. LNF was the most common surgical treatment. For small hiatal hernia repair, the well-documented benefits of LNF compared with open repair are many, including less postoperative pain, earlier discharge and return to work, and equal efficacy. 


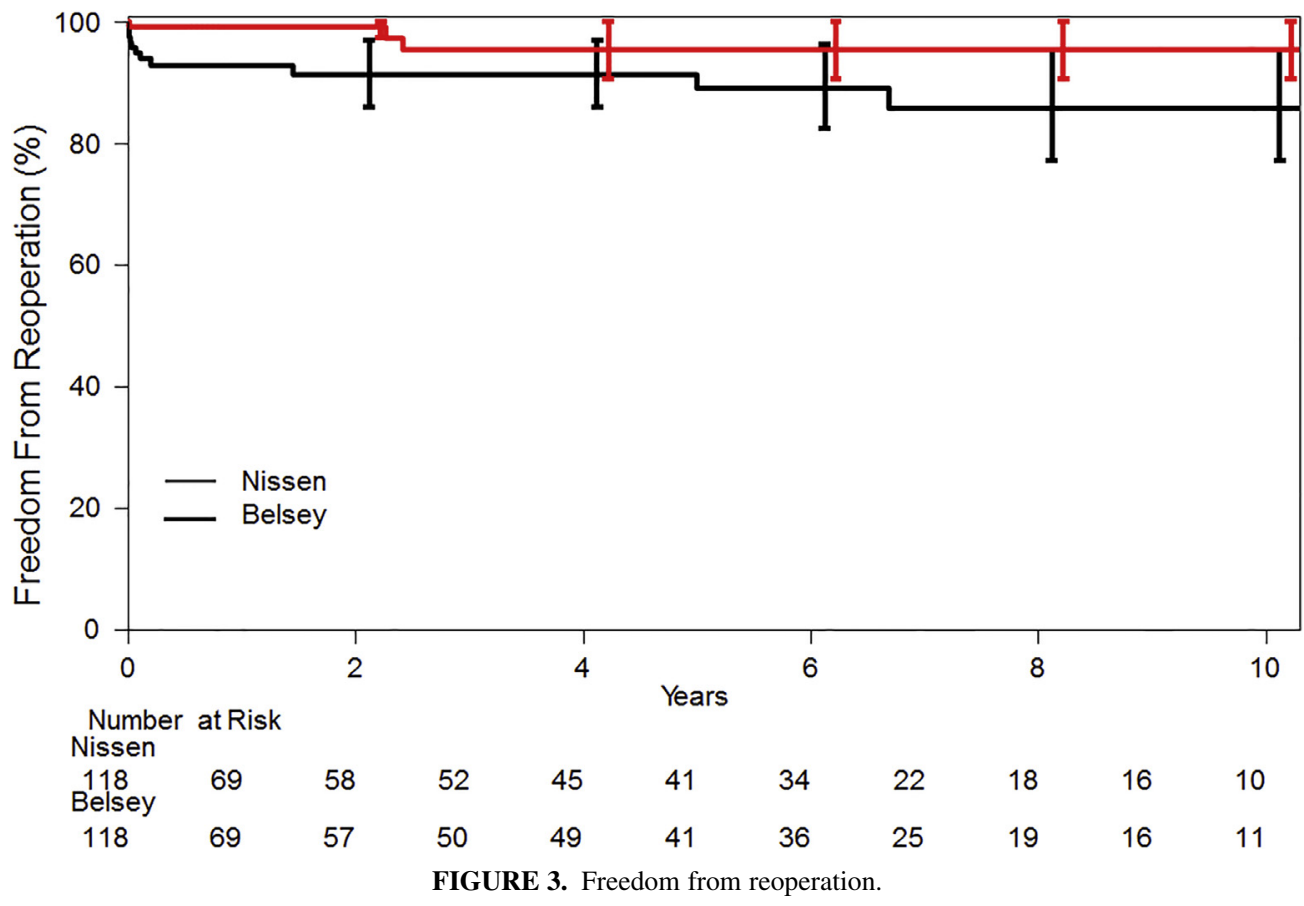

However, for patients with large paraesophageal hernias (those with $>50 \%$ of the stomach in the chest), we found LNF to be associated with a increased rate of leak and a higher rate of reoperation when compared with BM-IV. Recurrence rates (as measured objectively based on radiographic and endoscopic characteristics) were higher in the LNF group, but did not reach statistical significance. The difficulty of dissecting out a large hernia sac laparoscopically is evidenced by similar operating times between our 2 cohorts. Although one might expect the LNF to be a quicker operation, the arduous hiatal dissection required could have led to a heightened risk of injury to the stomach or esophagus, and be a cause of the increased rate of early postoperative leak and reoperation observed in the LNF cohort.

For smaller hernias, a minimally invasive approach seems to yield excellent results. This same trend may not be consistent in the face of an intrathoracic stomach. The question of abdominal versus transthoracic approach to hiatal hernia repair is debated in patients with large paraesophageal hernia. Transthoracic hiatal hernia repair has been advocated by Altorki and colleagues ${ }^{19}$ to permit adequate mobilization of esophagus to perform a tension-free and durable repair. LNF in such a context is associated with difficulty obtaining adequate crural closure, limited esophageal dissection within the mediastinum, and the need for wedge gastroplasties to lengthen the esophagus. The primary advantage of the BM-IV, distinguishing it from the LNF, is the ability to extensively dissect the mediastinal esophagus with limited damage to the crura or fundus. The noted benefits of a BM-IV repair are challenged by the increased morbidity of a thoracotomy, single lung isolation, and a longer length of stay.

The largest series of BM-IV repairs for large paraesophageal hernias was reported by Maziak and colleagues in 1998. ${ }^{15}$ In this series, 91 patients underwent BM-IV repairs, and 75 of those required esophageal lengthening via a Collis gastroplasty. There were 4 reported leaks and 2 deaths in that cohort. By comparison, none of the BM-IV cases in our retrospective series underwent an esophageal lengthening procedure, with comparable recurrence patterns and reoperation rates. Accordingly, esophageal lengthening procedures may predispose to postoperative leaks given the exposed staple line and extensive dissection. In the face of low long-term recurrence rates, the absence of leaks in our BM-IV cohort questions the need for esophageal lengthening when performing a transthoracic repair-in contrast to the high prevalence of Collis gastroplasty reported by Maziak and colleagues. ${ }^{15}$

Although laparoscopic hiatal hernia repair has been studied extensively, outcomes after laparoscopic repair of giant paraesophageal hernia are an evolving and distinct topic. The largest series to date was by Luketich and colleagues in $2010{ }^{3}$ This study showed an overall leak rate of $2.5 \%$, whereas our study showed a leak rate of $6.8 \%$. We matched the BM-IV group $(\mathrm{n}=118)$ with the LNF group $(\mathrm{n}=118)$, and in doing so we only examined a quarter $(n=118 / 442)$ of patients treated by LNF at our institution. The outcome of leak in this selected cohort may be greater than what would be observed in our total LNF cohort. Luketich and colleagues $^{3}$ observed a $4.9 \% 30$-day reoperation rate in patients treated with LNF, which is similar to our 90-day 
reoperation rate of $5.9 \%$ for patients receiving LNF. Of the patients with leak, $5(62.5 \%)$ had LNF with wedge gastroplasty and $3(37.5 \%)$ had LNF without wedge gastroplasty. The rate of esophageal lengthening procedures was as high as $84 \%$ initially in our study and could have contributed to high leak rate we observed. The rate of an esophageal lengthening procedure decreased to a nadir of $54 \%$ by the end of the study period, which is similar to other centers specializing in the treatment of giant paraesophageal hernia. ${ }^{3}$ The rate of leak was evenly distributed among surgeons' cases at our institution. Leaks occurred in patients undergoing operation by surgeons with greater than 20 years of experience and by younger surgeons with less than 10 years of experience as well. The total number of benign esophageal laparoscopic surgeries performed by surgeons in our group was stable throughout the study period and was roughly similar among surgeons.

Recurrence in patients treated with laparoscopic repair of large hiatal hernia is a debated topic; however, a recent meta-analysis by Rathore and colleagues ${ }^{4}$ reported a recurrence rate of $25 \%$ for patients with paraesophageal hernias treated with LNF, which is comparable to our recurrence rate in those treated with $\operatorname{LNF}(16.1 \%)$ in this same patient population. ${ }^{4}$ Rathore and colleagues ${ }^{4}$ found that the performance of a wedge gastroplasty was protective of recurrence, and we also observed a decreased recurrence in patients who had a wedge gastroplasty.

Increased intra-abdominal pressure as a result of central obesity predisposes obese patients to both GERD and hiatal hernia. ${ }^{20}$ The BM-IV is advantageous in obese patients in that abdominal adiposity is not encountered in the operation versus LNF. Median BMI was higher in the BM-IV group compared with the LNF group-a difference that would typically bias the results in favor of the LNF results (a finding not supported by our results). Patients who were more obese could have been preferentially selected for Belsey fundoplication given its advantage in the obese population. Reports of laparoscopic Roux-en-Y gastric bypass with hiatal closure have shown success in controlling GERD symptoms with the added advantage of subsequent weight $\operatorname{loss}^{21}$; however, the long-term durability of this repair when accompanied by large paraesophageal hernia has yet to be determined.

Length of stay and time to resumption of oral intake were less in the LNF group as expected. Although we might assume costs to be lower in the LNF group, as other reports have shown, ${ }^{2,22}$ this was not an end point of our study, and thus we did not collect financial data. The cost of repeat operation and management of gastrointestinal leaks should be factored into any cost comparison of the 2 procedures. There was no difference in the rate of postoperative complications score as defined by the CD scoring system; however, patterns of complications did differ in that leak was greater in the LNF group and that approximately half of patients who required a reoperation in the LNF group had the reoperation done so for the indication of leak (not symptomatic recurrence). Total reoperation rates were higher in the LNF cohort; however, reoperation specifically for symptomatic recurrence was approximately similar ( $\mathrm{n}=3$ in BM-IV, $\mathrm{n}=5$ in LNF) between groups. If the reoperations for leak are excluded, the need for reoperation is similar between the 2 groups. A greater percentage of patients in the BM-IV group had respiratory complications compared with LNF, but this did not reach statistical significance (Table 3). These differences in the types of complications should be considered when choosing which operative approach to offer a patient with a large hiatal hernia.

In their review of laparoscopic compared with open Nissen repair of large paraesophageal hernias, Hashemi and colleagues ${ }^{23}$ found an advantage of an open repair with regard to recurrence. Although we did not include open abdominal or transthoracic Nissen fundoplication in our analysis, it is plausible that these 2 open operations along with BM-IV may be similar in their efficacy to manage patients with large paraesophageal hernias. Further analysis is needed to delineate a tailored operative approach of the patient with large paraesophageal hernia.

\section{Study Limitations}

There are several limitations worth noting when evaluating the results of this study. First, selection bias in this study cannot be overlooked. Patients who were at higher risk of recurrence might have been selected for the BM-IV, whereas those at less risk might have had the LNF. This may indeed be true given differences in preoperative hernia size. All patients $(\mathrm{n}=118)$ in the BM-IV group had at least $75 \%$ of their stomach in the chest, whereas only $44 \%(n=53)$ of the LNF group had at least $75 \%$ of their stomach in the chest. The rest of the patients in the LNF group had moderate paraesophageal hernias $(>50 \%$, but $<75 \%$, of the stomach within the chest). However, given the differences in preoperative hernia size, the comparability of outcomes in the 2 groups comes into question. Second, only $45 \%$ of patients still alive at the time of this study responded to the GERD-HRQL questionnaire. The follow-up symptoms were by history only and were not verified by rigorous $\mathrm{pH}$ testing, EGD, and esophagrams in all patients. We did observe a difference in median clinical follow-up between the 2 groups: BM-IV $=657$ days (1.8 years), $\mathrm{LNF}=959$ days (2.6 years). However, when comparing symptoms scores assessed at the time of this study, median time from surgery to responding to the questionnaire was similar between groups.

Another limitation is the role of confounding bias related to surgeon preference. The main surgeon who performed BM-IV at our institution (M.S.A.) chooses not to perform LNF in 
patients with large paraesophageal hernias, but rather primarily selects the BM-IV as a matter of preference. As such, there are no comparative data in this cohort for LNF when M.S.A. was the surgeon. This creates the bias of comparing largely 1 surgeon's outcomes and experience with BM-IV with the rest of the surgeons in the group who primarily performed LNF. When BM-IV was performed by surgeons other than M.S.A., it was typically done so in patients with morbid obesity or a history of multiple previous abdominal operations, making abdominal dissection of the hiatus difficult.

\section{CONCLUSIONS}

LNF for large paraesophageal hernias was associated with a higher rate of leak and a higher rate of reoperation when compared with BM-IV. The findings of this comparative retrospective study highlight the value of the BM-IV as a viable treatment option when encountered by a patient with a large paraesophageal hernia. The decision as to which operative approach should be undertaken should take several patient-related and surgeon-specific factors into consideration. Overall, however, the surgeon should be aware of the advantages of a transthoracic approach in the carefully selected patient.

\section{Webcast}

You can watch a Webcast of this AATS meeting presentation by going to: https://aats.blob.core.windows.net/media/ 17AM/2017-05-01/BallroomABC/05-01-17_BallroomABC_ 0750_Laan.mp4.

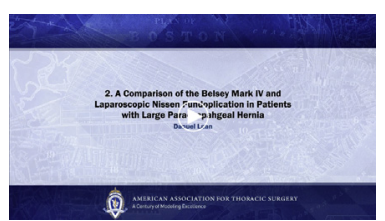

\section{Conflict of Interest Statement}

Authors have nothing to disclose with regard to commercial support.

\section{References}

1. Jensen CD, Gilliam AD, Horgan LF, Bawa S, Attwood SE. Day-case laparoscopic Nissen fundoplication. Surg Endosc. 2009;23:1745-9.

2. Richards KF, Fisher KS, Flores JH, Christensen BJ. Laparoscopic Nissen fundoplication: cost, morbidity, and outcome compared with open surgery. Surg Laparosc Endosc. 1996;6:140-3.

3. Luketich JD, Nason KS, Christie NA, Pennathur A, Jobe BA, Landreneau RJ, et al. Outcomes after a decade of laparoscopic giant paraesophageal hernia repair. J Thorac Cardiovasc Surg. 2010;139:395-404. 404.e1.

4. Rathore MA, Andrabi SIH, Bhatti MI, Najfi SMH, McMurray A. Metaanalysis of recurrence after laparoscopic repair of paraesophageal hernia. JSLS. 2011;11: 456-60.

5. Nissen R. Reminiscences-reflux esophagitis and hiatal hernia. Rev Surg. 1970; 27:307-14.

6. Nissen R. A simple operation for control of reflux esophagitis. Schweiz Med Wochenschr. 1956;86:590-2.
7. Donahue PE, Larson GM, Stewardson RH, Bombeck CT. Floppy Nissen fundoplication. Rev Surg. 1977;34:223-4.

8. DeMeester TR, Bonavina L, Albertucci M. Nissen fundoplication for gastroesophageal reflux disease. Evaluation of primary repair in 100 consecutive patients. Ann Surg. 1986;204:9-20.

9. Pearson FG, Henderson RD. Long-term follow-up of peptic strictures managed by dilatation, modified Collis gastroplasty, and Belsey hiatus hernia repair. Surgery. 1976;80:396-404.

10. Hiebert CA. Surgical management of esophageal reflux and hiatal hernia. Ann Thorac Surg. 1991;52:159-60.

11. Stylopoulos N, Rattner DW. The history of hiatal hernia surgery: from Bowditch to laparoscopy. Ann Surg. 2005;241:185-93.

12. Coosemans W, De Leyn P, Deneffe G, Van Raemdonck D, Lerut T. Laparoscopic antireflux surgery and the thoracic surgeon: what now? Eur J Cardiothorac Surg. 1997; 12:683-8.

13. Menguy R. Surgical management of large paraesophageal hernia with complete intrathoracic stomach. World J Surg. 1988;12:415-22.

14. Wichterman K, Geha AS, Cahow CE, Baue AE. Giant paraesophageal hiatus hernia with intrathoracic stomach and colon: the case for early repair. Surgery. 1979; 86:497-506.

15. Maziak DE, Todd TRJ, Pearson FG. Massive hiatus hernia: evaluation and surgical management. J Thorac Cardiovasc Surg. 1998;115:53-62.

16. Allen MS, Trastek VF, Deschamps C, Pairolero PC, Ellis FH Jr, Connolly JE. Intrathoracic stomach: presentation and results of operation. J Thorac Cardiovasc Surg. 1993;105:253-9.

17. Velanovich V. The development of the GERD-HRQL symptom severity instrument. Dis Esophagus. 2007;20:130-4.

18. Dindo D, Demartines N, Clavien P-A. Classification of surgical complications: a new proposal with evaluation in a cohort of 6336 patients and results of a survey. Ann Surg. 2004;240:205-13.

19. Altorki NK, Yankelevitz D, Skinner DB. Massive hiatal hernias: the anatomic basis of repair. J Thorac Cardiovasc Surg. 1998;115:828-35.

20. Frezza EE, Shebani KO, Robertson J, Wachtel MS. Morbid obesity causes chronic increase of intraabdominal pressure. Dig Dis Sci. 2007; 52:1038-41.

21. Perry Y, Courcoulas AP, Fernando HC, Buenaventura PO, McCaughan JS, Luketich JD. Laparoscopic Roux-en-Y gastric bypass for recalcitrant gastroesophageal reflux disease in morbidly obese patients. JSLS J Soc Laparoendosc Surg. 2004;8:19-23.

22. Laycock WS, Oddsdottir M, Franco A, Mansour K, Hunter JG. Laparoscopic Nissen fundoplication is less expensive than open Belsey Mark IV. Surg Endosc. 1995;9:426-30.

23. Hashemi M, Peters JH, DeMeester TR, Huprich JE, Quek M, Hagen JA, et al. Laparoscopic repair of large type III hiatal hernia: objective followup reveals high recurrence rate. J Am Coll Surg. 2000;190:553-60.

24. Sami S, Ragunath K. The Los Angeles classification of gastroesophageal reflux disease. Video J Encycl GI Endosc. 2013;1:103-4.

Key Words: Belsey Mark IV, laparoscopic Nissen fundoplication, paraesophageal hernia

\section{Discussion}

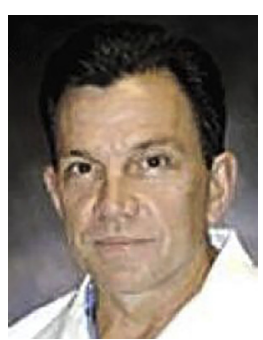

Dr J. Luketich (Pittsburgh, Pa). Your article indicates that 1 surgeon performed $94 \%$ of the Belsey operations, whereas the lap Nissen was distributed more evenly among multiple surgeons. Do you think the difference in outcome was simply a factor of surgeon experience and learning curves?

If one considers that the Belsey is a better operation, has this convinced any of your surgeons to abandon lap Nissen in favor of open Belsey, and do you think patients will 
accept this or go elsewhere to seek minimally invasive options as history would strongly suggest?

At the American Association for Thoracic Surgery 2 decades ago, Dr Maziak presented Dr Pearson's long-term results of the Belsey done open with a Collis gastroplasty. Dr Pearson performed most of these operations as well. In this study, the main follow-up was over 6 years with only 2 reoperations. With your follow-up duration being somewhat less, at 3 years, do you think your recurrence rates will continue to increase over time and was the exclusion of the Collis potentially a contributing factor?

In your longer follow-up subset, you show that the postoperative symptom scores were excellent in approximately $80 \%$ of patients, which was an improvement over those with less follow-up. This is somewhat the opposite of most studies of laparoscopic or open repair of antireflux operations. Do you have any explanation why the results actually got better over time?

In our own extensive experience with lap giant hernia repairs, we found that after over a decade of experience we were able to get our recurrence rates down to $3 \%$ at a median follow-up of over 7 years. However, again, most were done by a single surgeon with only 2 other surgeons contributing, but each of them had in excess of 50 cases. Even now, our lower-volume surgeons will seek a highervolume experienced colleague to help them. Have you considered this?

The lap giant paraesophageal hernia has several important components as does the open. In your series were all the steps standardized, that is, does everyone do it the same, and are there any specific credentialing criteria, that is, minimum number of cases or outcomes, or can anyone book this case open or laparoscopic?

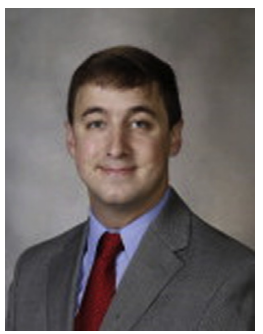

Dr Danuel Laan (Rochester, Minn). We do acknowledge the limitation of comparing largely 1 surgeon's experience with multiple surgeons; however, we also note that our laparoscopic surgeons at the Mayo Clinic have sufficient expertise and high volume in benign esophageal laparoscopic surgery. The rate of leak was evenly distributed among surgeons at our institution. Leaks occurred in patients undergoing operation by surgeons with greater than 20 years of experience and by younger surgeons with less than 10 years of experience as well. The total number of benign esophageal laparoscopic surgeries performed by surgeons in our group was stable throughout the study period and was roughly similar among surgeons, making it unlikely that an expertise bias is confounding the results of our study.

With regard to your second and third questions, we believe that the results of this study potentially highlight the need of the Belsey fundoplication in the armamentarium of the thoracic surgeon. More study in a prospectively randomized control fashion will be needed to further delineate the advantages and disadvantages of both of these operations.

Concerning the Maziak and Pearson study in 1997, we acknowledge the contributions of Drs Maziak and Pearson. They had excellent long-term follow-up and a low number of recurrences. It is possible that with longer follow-up, we may observe more recurrences in our Belsey cohort. An important difference between our study and the Maziak and Pearson study is the use of an esophageal lengthening procedure. In their study, the majority of patients had an esophageal lengthening procedure; in our study, zero of our Belsey cases had an esophageal lengthening procedure. The rate of leak in their study was approximately $5 \%$; in our study, there were no leaks in the Belsey fundoplication cohort. I think this brings into question the necessity of performing an esophageal lengthening procedure in a Belsey fundoplication, especially given the potential added risk.

With regard to our original symptomatic follow-up data, which were based strictly on clinical follow-up with a provider, we followed only 41 patients for more than 5 years, so drawing long-term conclusions from that is difficult. As such, we attempted to contact all study patients by phone and mail out surveys to obtain a current symptoms score using the GERD-HRQL Questionnaire to obtain an accurate assessment of their current symptoms. We were able to reach approximately half of patients still living at the time of this study and found no difference in symptoms score, use of acid-reducing medication, or degree of satisfaction between groups. Given that our original symptomatic follow-up was strictly based on review of the chart from follow-up appointments, we have removed these data from the article and replaced it with the more granular GERD-HRQL Questionnaire data.

With regard to standardization of practice among our laparoscopic surgeons. We believe that all of our surgeons focus on hiatal hernia sac excision, obtaining an adequate length of intra-abdominal esophagus, and the addition of an esophageal lengthening procedure only when necessary. We did not observe that any 1 surgeon contributed to the rate of complication or recurrence more so than any other surgeon. Again, I think this represents the low likelihood that an expertise bias is contributing as a confounder to our results.

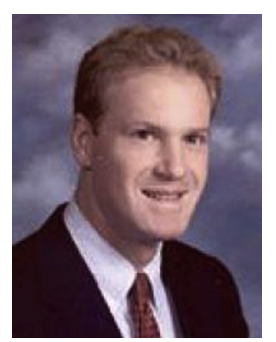

Dr S. DeMeester (Portland, Ore). A very nice series and contribution, particularly on your transthoracic results. One question on your methodology. You defined a recurrence as physiologic. I am not sure what a physiologic recurrence is. Most people use an objective criterion, either an endoscopic finding of recurrent hernia or a measured size on a barium swallow. Can you clarify that for me, and did these 
patients get objective follow-up in recurrence or is this symptomatic recurrence only?

The second issue is in the leaks, if you could clarify that, because a Nissen doesn't leak. So that would imply there was an esophageal injury during the dissection, and we have learned a lot in the laparoscopic management of these giant hernias over the last 2 decades. So were these early injuries happening early in the experience with an esophageal injury taking down the hernia sac, because otherwise how does a Nissen leak? You don't open the gastrointestinal tract.

Dr Laan. With regard to your first question, hernia recurrence was defined completely as a radiologic or endoscopic finding. Recurrence was noted if the study demonstrated greater than physiologic herniation of the gastroesophageal junction above the diaphragmatic hiatus $(>2 \mathrm{~cm})$, a slipped wrap, or a disrupted wrap. Expected postwedge gastroplasty radiographic or endoscopic features were not reported as recurrences.

With regard to your question concerning leak, we believe that the arduous hiatal dissection required to adequately mobilize the esophagus is a contributing factor for the increased rate of leak we observed in the LNF cohort. The addition of an esophageal lengthening procedure to LNF may also contribute. The Belsey fundoplication is advantageous in its ability to dissect out the esophagus with limited damage to the cura and fundus.

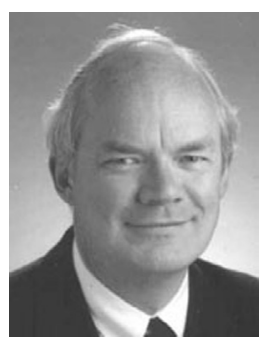

Dr R. Finley (Vancouver, British Columbia, Canada). Again, congratulations on following up, particularly the Belsey. I was a little concerned about your leak rate. I think if I was a patient and came to you and you said you had a 1 in 15 chance of leaking, it would be of concern to me. The Belsey is a partial fundoplication; the Nissen is a complete fundoplication. Were your complications related to bloating or not?

Second, with the thoracotomy, were there any long-term pain problems in relationship to the thoracotomy and Belsey? Specifically, what have you done to try to reduce this leak rate at which you had 1 fatality?

Dr Laan. With regard to your first question, we believe that the leak rate may be underrecognized within LNF of large hiatal hernias. As a tertiary referral center, we have seen a number of referrals from outside hospitals for leak in the setting of LNF. Second, dysphagia as a specific symptom was not different between groups. Third, we did evaluate patients for post-thoracotomy pain syndrome. There was 1 patient in the Belsey group who required a pain specialty consult for post-thoracotomy pain, and I think that is one of the risks of the operation that needs to be discussed with the patient.

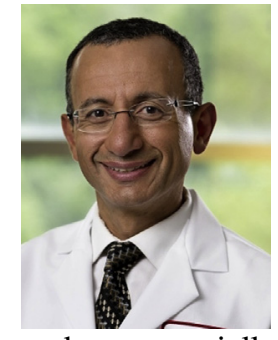

Dr A. Abbas (Philadelphia, Pa). Congratulations on an excellent presentation and to your group for tackling a difficult problem. One comment is that letting the surgeons decide what percent of the stomach is actually in the chest is a little like asking a fisherman how big the fish he caught was. It is very difficult to do so, especially if you are doing it through a thoracotomy. The question I have is related to your results. What do you think the mechanism of superiority of a Belsey over a Nissen is? At the end of the day, a hiatal hernia repair is an operation for repair of the diaphragm. Do you think that doing it from the chest is simply a better way to do that repair?

Dr Laan. We recognized the possible subjectivity in defining hernia size based on operative findings. Second, with regard to the mechanism of possible superiority, we believe that the Belsey fundoplication, a transthoracic repair, offers superior mobilization of the esophagus up to the level of the aortic arch with limited damage to the esophagus, cura, and fundus, and we believe our data support that.

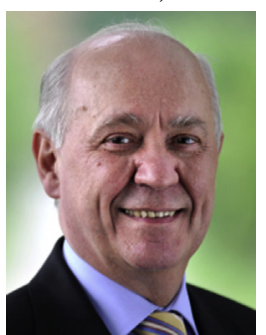

Dr T. Lerut (Leuven, Belgium). Actually, we also have the same results in the treatment of these giant paraesophageal hernias. Having had the privilege to be Belsey's last pupil, I know by experience that this is not an easy operation and certainly not an easy operation to teach. You can't do it thoracoscopically; it is simply impossible. And as has been pointed out, I think the actual experience is still in the hands of those surgeons who were familiar with the BM-IV before the laparoscopic era.

So, given your results that it should stay within the training armamentarium of the thoracic surgeon, how can you give your possibility to bring this into the training program of thoracic surgeons of the future?

Dr Laan. Your question highlights the overall point of our study in that the Belsey fundoplication needs to be maintained in the armamentarium of the thoracic surgeon. We hope this presentation and subsequent publication will increase awareness of the benefits of transthoracic repair of large paraesophageal hernias. There is a article by Dr Allen, "The Belsey Procedure, How I Teach It," which elucidates his teaching points on the procedure. This article was of great help to me in learning the pearls and pitfalls of the Belsey procedure.

Dr Moon. Dr Luketich, final word.

Dr Luketich. In closing, I believe there is, no doubt, much to be learned from our open surgical history and from our senior surgeons, of which I consider myself one. But I do not believe our patients will accept a backward progress to open surgery. For thoracic surgeons to retain control of this patient population and others, we have to improve on our minimally invasive results. 